\title{
Design Methodology for Reducing RIN Level in DFB Lasers
}

\author{
Hisham K. Hisham \\ Electrical Engineering Department, Engineering College, \\ University of Basrah, \\ Basra, Iraq \\ E-mail: husham_kadhum@yahoo.com
}

\begin{abstract}
The relative intensity noise (RIN) characteristics in distributed feedback (DFB) lasers are analyzed theoretically by proposing a new methodology. In addition to temperature variation (T), the effect of other model parameters such as injection current $\left(I_{i n j}\right)$, active layer volume $(V)$, spontaneous emission $\left(\beta_{s p}\right)$ and gain compression (ع) factors on RIN characteristics is investigated. The numerical simulations shows, the peak RIN level can be reduced to around $-150 \mathrm{~dB} / \mathrm{Hz}$, while relaxation oscillation frequency $(\mathrm{ROF})$ is shifted towards $5.6 \mathrm{GHz}$. In addition, the RIN level is increased with temperature by the rate of $0.2 \mathrm{~dB} /{ }^{\circ} \mathrm{C}$ and ROF is reduced by the rate of $0.018 \mathrm{GHz} /{ }^{\circ} \mathrm{C}$. Results show, the low RIN level can be obtained by selecting model parameters reasonably.
\end{abstract}

Index Terms- Distributed feedback (DFB) lasers, relative intensity noise (RIN), semiconductor diode lasers (SDLs).

\section{INTRODUCTION}

With the huge increase in demand for high-speed fiber optic internet services, the need to systems that support large data transfer became an essential [1]. Up to now, wavelength division multiplexing (WDM) systems with up to $50-\mathrm{GHz}$ channel spacing have already been reported [2]. However, further progress in the field of information technology makes the need to increase the ability to transfer data to levels much higher is absolutely imperative [3]. To achieve this, WDM systems with laser source with very high wavelength stability, low static and dynamic chirp and with reasonable cost is required [4].

Distributed feedback (DFB) lasers are considered as an effective and reliable light source for WDM systems due to their good dynamic single-mode (DSM) performance, larger side-mode suppression ratio (SMSR), compact size, integration capability, etc.

However, low cost DFB lasers with an excellent performance are still difficult to achieve, due to highly dependence for their emission wavelength on the injected current and temperature variations [5].
On the other hand, DFB lasers are intrinsically noisy device due to the quantum nature of the light. The fluctuations in the light intensity are directly effect on the lasers performance, and it is characterized by the relative intensity noise (RIN) [6]. The effect of RIN level on the performance of DFB lasers have been investigated in several numerical and experimental studies [5, 6-11]. In 2006, I. Fatadin et al. [7] have investigated the effect of injected current on the RIN characteristics and they show that by injecting current of $26.3 \mathrm{~mA}$, the peak RIN level can be reduced to about $-135 \mathrm{~dB} / \mathrm{Hz}$. In 2012, $\mathrm{M}$. Faugeron et al. [11] have designed a DFB laser operating with a RIN level of $-157 \mathrm{~dB} / \mathrm{Hz}$. Also, in $2013 \mathrm{M}$. Faugeron et al. [10] have developed a DFB laser operating with a RIN level of -160 $\mathrm{dB} / \mathrm{Hz}$. While, in 2015; J. Liu et al. [9] have reduced the RIN level for the DFB laser to -165 $\mathrm{dB} / \mathrm{Hz}$. However, all these studies have lacked to accuracy due to ignore the effect of other affected laser parameters; where it did not take into account the effect of temperature variations, cavity volume, spontaneous emission factor and gain compression factor. Therefore, the results 
obtained do not reflect the true reality of the performance of these lasers.

In this paper, we present for the first time to our knowledge, a comprehensive study on the reducing RIN level of DFB laser. We modeled the temperature dependence (TD) of threshold carrier density $\left(N_{t h}\right)$ according to the TD of laser cavity parameters. Furthermore, unlike all the previous studies; the effect of temperature variations on RIN characteristics has calculated according to TD of laser parameters instead of well-known Pankove equation.

The paper is structured as follows: the design methodology is given in the next section. Section 3 presents the theoretical model for DFB laser. In Sect. 4, we demonstrate the lasers rate equations with Langevin noise sources. The simulation results are discussed in Sect. 5 followed by the conclusions.

\section{DESIGN METHODOLOGY}

For reducing the RIN level in DFB lasers, we proposed a new methodology (as shown in Fig. 1) based on investigate the effect of each affected parameters separately in order to get the optimal design. The process begins with the DFB laser model. In the next step, all the expressions that describe the DFB performance are modified to include the effect of temperature variations. By referring to Fig. 1, if all the analyzed results are accurate (i.e. if RIN level within the acceptable limits), the methodology flow proceeds to the next step. If not, methodology flow will be repeated again. Finally, the flow will be completed if the analyzed results are correct.

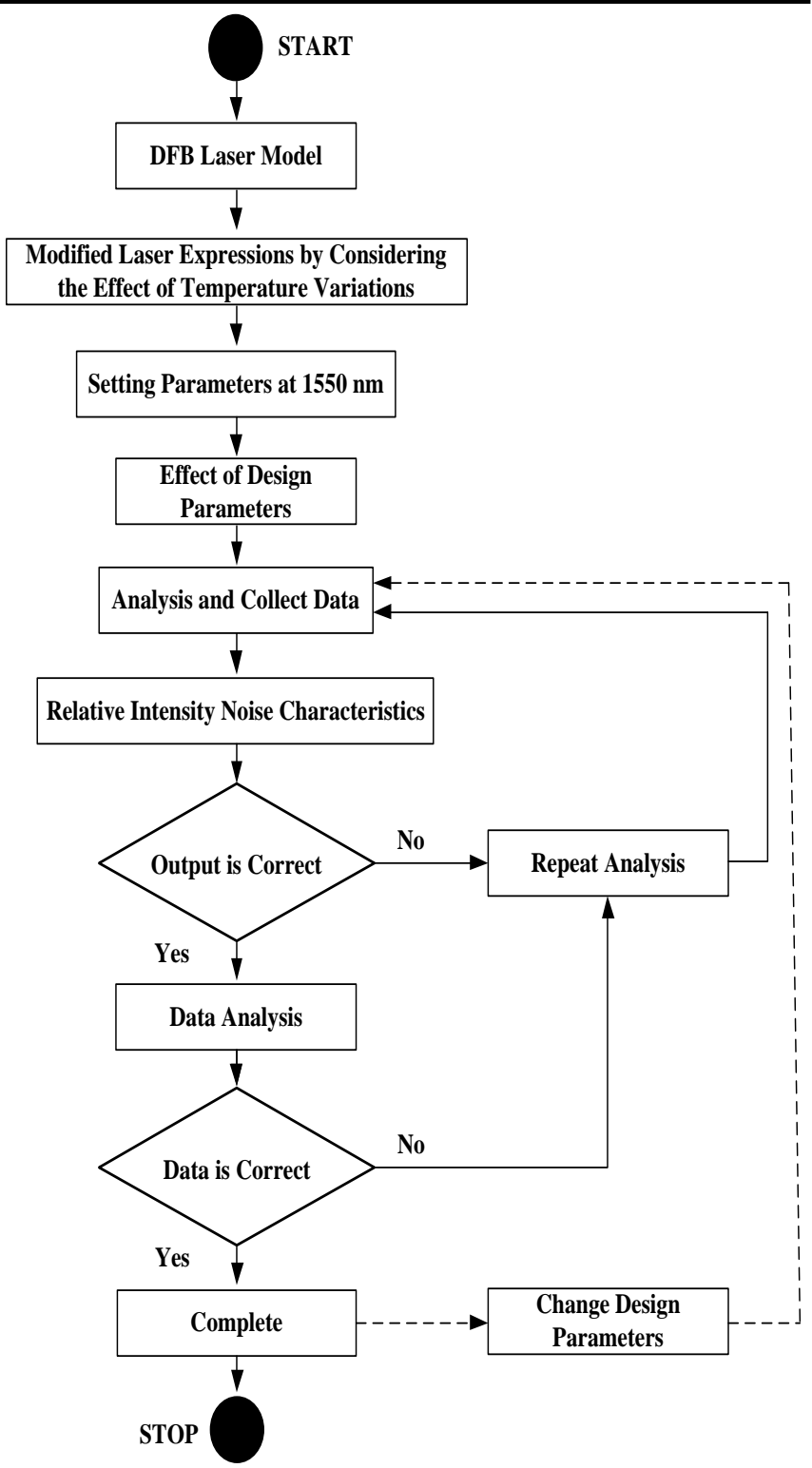

Fig. 1 The Methodology Flow for reducing the RIN level in DFB lasers

\section{THEORTICAL MODEL}

By considering the effect of the temperature $(T)$ variations, the DFB laser threshold current can be defined as [12]

$I_{t h}(T)=q V N_{t h}(T) \Psi\left(T, N_{t h}\right)$

In Eq. (1), $q$ is the electron charge, $V$ is the volume of the DFB active layer region, and $\Psi(T$, $\left.N_{t h}\right)$ is the modified carriers recombination rate coefficient [13], which can be rewritten as

$\Psi\left(T, N_{t h}\right)=A_{n r}+B N_{t h}(T)+C(T) N_{t h}^{2}(T)$ 
where $A_{n r}$ and $\mathrm{C}(T)$ describe the nonradiative recombination rate and TD Auger process, respectively, while $B$ is the radiaitive recombination coefficient. The $N_{t h}(T)$ in Eq. (2) is the well-known TD carrier density at threshold condition [12], in which by considering the effect of temperature $(T)$ variations, can be defined as

$$
N_{t h}(T)=N_{o}(T)+\frac{1}{\Gamma v_{g}(T) a(T) \tau_{p}(T)}
$$

where $N_{o}(T), a(T), \quad v_{g}(T)$ and $\tau_{p}(T)$ are the temperature dependence transparency carrier density, gain constant, and photon life time, respectively. In Eq. (3), $\tau_{p}(T)$ can be modelled as $[12,13]$

$\tau_{p}(T)=\frac{1}{v_{g}(T) \alpha_{t o t}(T)}$

where $\alpha_{t o t}(T)$ is the temperature dependence total cavity loss that is defined as [12]

$$
\alpha_{t o t}(T)=\alpha_{i n t}(T)+2 \alpha_{o}
$$

where $\alpha_{i n t}(T)$ is the internal cavity loss, and $\alpha_{o}$ is the threshold gain of the main mode. Finally, the $N_{t h}(T)$ can be expressed as

$$
N_{t h}(T)=N_{o}(T)+\frac{\alpha_{i n t}(T)+2 \alpha_{o}}{\Gamma a(T)}
$$

Equation (6) gives general expression that can be used to calculate the TD threshold carrier density. In this study, the TD of the model is assumed vary according to [6].

\section{LASER RATE EQUATIONS with LANGEVIN NOISE SOURCES}

By considering the effect of temperature $(T)$ variation, the dynamics characteristics of DFB lasers can be derived from the well-known coupled rate equations $[12,13]$. Relationship between noise sources, $F_{i}(t)$, carrier number $N(t)$, photon number $P(t)$, and optical phase $\phi(t)$ is described as [7]

$$
\frac{d N(t)}{d t}=\frac{I(t)}{q}-\frac{N(t)}{\tau_{c}}-g \frac{N(t)-N_{o}}{1+\varepsilon P(t)} P(t)+F_{N}(t)
$$

$\frac{d P(t)}{d t}=g \frac{N(t)-N_{o}}{1+\varepsilon P(t)} P(t)-\frac{P(t)}{\tau_{p}}+R_{s p}+F_{P}(t)$

$\frac{d \phi(t)}{d t}=\frac{\alpha}{2} g(N(t)-\bar{N})+F_{\phi}(t)$

where, $I(t)$ is the injected current, $\tau_{c}=q V N_{t h} / I_{t h}$ is the carrier lifetime, $g$ is the gain slope constant coefficient, $\varepsilon$ is the nonlinear gain compression factor, $R_{s p}$ represents the contribution of the spontaneous emission of the lasing mode, $\alpha$ is the linewidth enhancement factor and $\bar{N}$ is the time-average carrier number. In Eq. (7), the term $(1+\varepsilon P(t))$ is assumed as an optical gain compression, which is may also appear in form of $(1-\varepsilon P(t))$ or $1 /(1-\varepsilon P(t))$. However, the formula in Eq. (7) fit dramatically with the numerical solutions [7].

For noise calculations, the fluctuations of the variables are assumed small for all times (smallsignal approximation). Under this assumption, Eq. (7) can be easily solved in the frequency domain. In Eq. (7), the random functions $F_{N}(t), F_{P}(t)$, and $F_{\phi}(t)$ are assumed the Langevin noise sources due to the carriers, photons, and phase, respectively. Based on the Markovian assumption, the relations between the noise sources can be represent as [13]

$$
\left\langle F_{i}(t)\right\rangle=0 \text { and }\left\langle F_{i}(t) \cdot F_{j}\left(t^{\prime}\right)\right\rangle=2 H_{i j} \delta\left(t-t^{\prime}\right)
$$

Where $\delta$ is the Dirac's delta function and $H_{i j}$ is the diffusion coefficients defined as $[8,13]$

$$
\begin{aligned}
& H_{P P}=\left[\frac{a \Omega}{V}\left(N+N_{o}\right)+G_{t h}\right] P+\frac{a \Omega N}{V} \\
& H_{N N}=\frac{a \Omega}{V}\left(N+N_{o}\right) P+\frac{N}{\tau_{c}}+\frac{I}{c} \\
& H_{\phi \phi}=\frac{H_{P P}}{4(P+1)^{2}}
\end{aligned}
$$

$D_{P N}=-\frac{H_{P P}}{2}$

$D_{S \phi}=D_{N \phi}=\frac{H_{P N}}{2(P+1)}$ 
In Eq. (9), $\frac{a \Omega N}{V}, \frac{a \Omega N_{o}}{V}, P$ and $N$ are represents the optical emission, optical absorption, steadystate values of the photon and carrier populations, respectively.

\section{RELATIVE INTENSITY NOISE in DFB LASERS}

Under small signal analysis for the linear set of Eq. (7), the RIN characteristics for the DFB lasers is defined by [13]

$R I N(\omega)=\frac{U Y^{2}\left(1+Z \omega^{2}\right)}{Y^{2}+\left(X^{2}-2 Y\right) \omega^{2}+\omega^{4}}$

Where the parameters $X, Y, Z$ and $U$ are functions of the DFB laser injection current $\left(I_{i n j}\right)$ and other model parameters, which given as [8]

$X=\frac{1}{\tau_{c}}+\frac{1}{\tau_{P}}+\frac{a}{(1+\varepsilon P)}\left[P-\frac{\left(N-N_{o}\right)}{1+\varepsilon P}\right]$

$Y=\frac{1}{\tau_{c} \tau_{P}}+\frac{a}{(1+\varepsilon P)}\left[\frac{P}{\tau_{P}}-\frac{\left(1-\beta_{f r c}\right)}{\tau_{c}}\left(\frac{N-N_{o}}{1+\varepsilon P}\right)\right]$

$Z=\left\{\frac{1}{\tau_{c}^{2}}\left[\left(1-\beta_{f}\right)^{2}+\frac{\beta_{f}}{P}\right]+\frac{a}{(1+\varepsilon P)}\left[\frac{2}{\tau_{c}}+\frac{P}{\beta_{f}}\left(\frac{a}{1+\varepsilon P}\right)\right]\right\}$

$U=\frac{2(1+\varepsilon P)^{2} N}{\alpha_{t o t}^{2}}\left[\frac{\beta_{f}\left(P\left(1-\beta_{f}\right)^{2}+\beta_{f}\right)(1+\varepsilon P)^{2}+a P \tau_{c}\left(a P \tau_{c}+2 \beta_{f} \varepsilon P+2 \beta_{f}\right)}{P^{2} \tau_{c}\left(\tau_{P} a\left(1-\beta_{f}\right)\left(N-N_{o}\right)-(1+\varepsilon P)\left(\left(a P \tau_{c}+(1+\varepsilon P)\right)\right)\right)^{2}}\right.$

Where $\beta_{f}$ represent the fraction of spontaneous emission coupled to the lasing mode, $Y$ and $Z$ are the damping factor and the relaxation oscillation frequency (ROF) of the DFB laser, respectively.
VI. RESULTS and DISCUSSION

The parameters are used in the simulation shown in Table I. All these values are fixed throughout this paper, except otherwise is stated.

TABLE I

DEVICE STRUCTURE AND MATERIAL PARAMETERS USED IN CALCULATIONS

\begin{tabular}{|l|l|}
\hline \multicolumn{1}{|c|}{ DFB Parameters } & \multicolumn{1}{c|}{ Description } \\
\hline$L=400 \mu \mathrm{m}$ & Cavity layer length \\
\hline$A=24 \times 10^{10} \mathrm{~m}^{2}$ & Active layer cross-sectional area \\
\hline$V=1.2 \times 10^{-16} \mathrm{~m}^{3}$ & Active layer volume \\
\hline$N_{o}=1^{\times} 10^{24} \mathrm{~m}^{-3}$ & Transparency carrier density \\
\hline$A_{n r}=1^{\times} 10^{8} \mathrm{sec}^{-1}$ & $\begin{array}{l}\text { Nonradiative recombination } \\
\text { coefficient }\end{array}$ \\
\hline$B=1^{\times} 10^{-16} \mathrm{~m}^{3} / \mathrm{sec}$ & $\begin{array}{l}\text { Radiative recombination } \\
\text { coefficient }\end{array}$ \\
\hline$C=3^{\times} 10^{-41} \mathrm{~m}^{6} / \mathrm{sec}$ & Auger recombination coefficient \\
\hline$\alpha_{\text {int }}=1000 \mathrm{~m}^{-1}$ & Internal cavity loss \\
\hline$\Gamma=0.34$ & Field confinement factor \\
\hline$R=0.56$ & $\begin{array}{l}\text { Reflectivity of the light inside } \\
\text { cavity }\end{array}$ \\
\hline$\varepsilon=1^{\times} 10^{-17} \mathrm{~cm}^{3}$ & Gain comparison factor \\
\hline$g=3.6^{\times} 10^{3} \mathrm{~cm}^{3} / \mathrm{sec}$ & Gain slope constant coefficient \\
\hline$a_{o}=2.5^{\times} 10^{-20} \mathrm{~m}^{2}$ & Differential gain \\
\hline$\alpha=4$ & $\begin{array}{l}\text { Linewidth enhancement } \\
\text { coefficient }\end{array}$ \\
\hline$I_{\text {inj }}=2 I_{t h}$ & Injection current \\
\hline
\end{tabular}

Figure 2 show the effect of laser injected current ( $I_{i n j}$ ) on the RIN characteristics. Result shown, by increasing $I_{i n j}$ from $1.3 I_{\text {th }}$ to $4 I_{t h}$, the peak RIN level has reduced from -107.54 to $-146.26 \mathrm{~dB} / \mathrm{Hz}$ and ROF has shifted toward higher frequencies from 0.5 to $3.8 \mathrm{GHz}$, thereby, a larger flat frequency range is obtained. Where, by increasing the $I_{i n j}$ value; the photons inside the active region have increased dramatically; which leads to reduce the fluctuations for the gain spectrum, and then reducing in the RIN level. From the other hand, ROF depends strongly on the $I_{i n j}$ value [ 8 13], and this explain why it shifted toward higher frequency. 


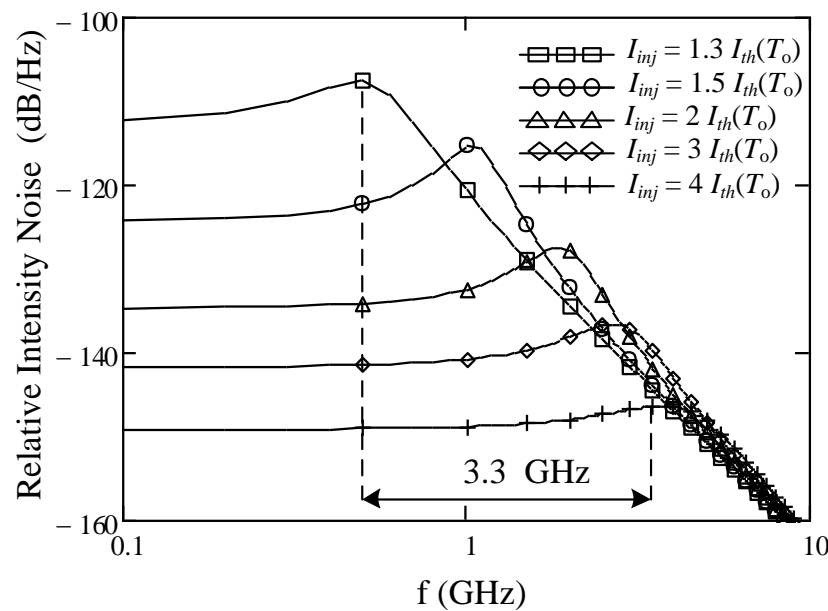

Fig. 2 Effect of laser injection current $\left(I_{i n j}\right)$ on the DFB RIN spectra

Figure 3 shown the effect of temperature variations on the RIN characteristics at $I_{i n j}=2 I_{t h}$. As can be seen from Fig. 3, by increasing temperature from 25 to $85^{\circ} \mathrm{C}$, the peak RIN level has increased from $\quad-127.6$ to $-115.5 \mathrm{~dB} / \mathrm{Hz}$ (i.e. increased by the rate of $0.2 \mathrm{~dB} /{ }^{\circ} \mathrm{C}$ ) and $\mathrm{ROF}$ has shifted back from 2 to $0.9 \mathrm{GHz}$ (i.e. reduced by the rate of $0.018 \mathrm{GHz} /{ }^{\circ} \mathrm{C}$ ). This is due to the highly dependence for the DFB wavelength on temperature. Where, temperature variations lead to shift the lasing frequency, which resulting in multi fluctuations inside the optical cavity. The shift in the operating frequency leads to reduce the output power; and thus increasing the RIN level. However, temperature effect can be reduced significantly by increasing the $I_{i n j}$ value as shown in Fig. 4.

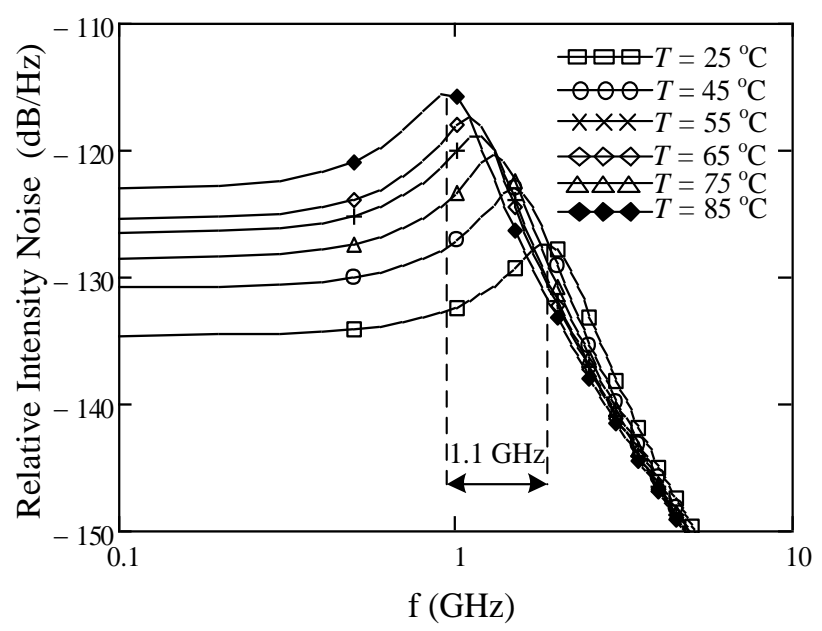

Fig. 3 Effect of temperature variation on DFB RIN spectra at $I_{i n j}=2 I_{t h}$

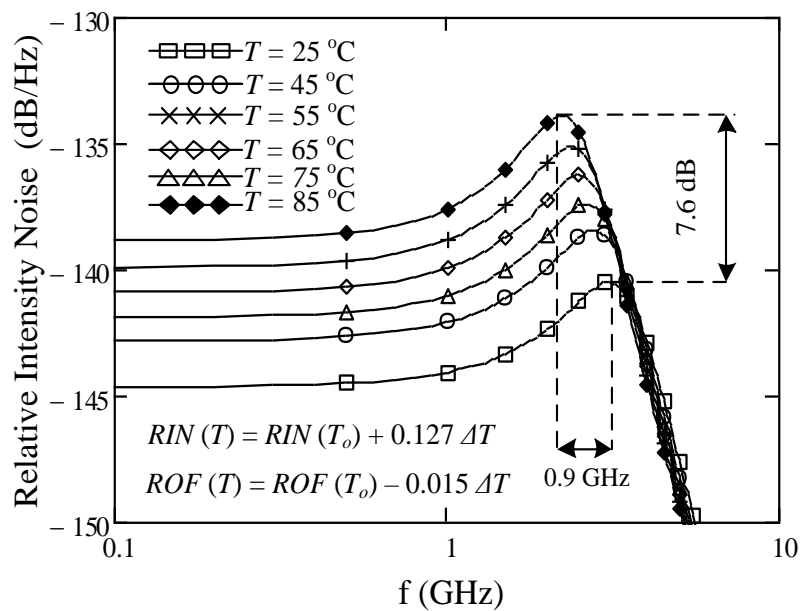

Fig. 4 Effect of temperature variation on DFB RIN spectra at $I_{i n j}=3 I_{\text {th }}$

For all laser designers, active-layer cavity volume represent a critical factor for the laser performance; where by reducing it may leads to increase the temperature effect. From the other hand, the large cavity volume leads to increase the total cavity loss. Therefore, it is necessary to find the optimal design for acceptable performance. Figure 5 show the effect of activelayer cavity volume $(V)$ on RIN characteristics. As can be seen by increasing $V$ value from $1.2 \times 10^{-10}$ to $2.8 \times 10^{-10} \mathrm{~cm}^{3}$ (through increasing any one of the $V$ parameters (i.e. active-layer cavity length, width and thickness), the ROF has shifted back toward lower frequency by about 3.1 $\mathrm{GHz}$ and the peak RIN level has increased by around $30 \mathrm{~dB}$. This results support the importance of finding the optimal design, where, it confirm the advantages of the smaller volume for reducing the total cavity loss and on the other hand, further reduction may lead to increase the thermal effect. In addition, further reduction in the active layer length results in short time delay, which may push the laser to operate at the transient region or chaotic dynamics [14]. 


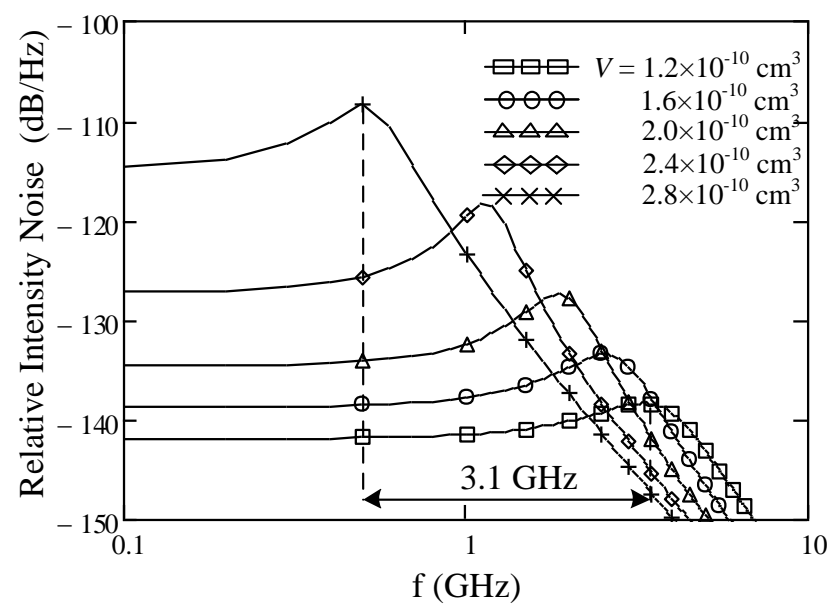

Fig. 5 Effect of cavity volume on the DFB RIN spectra

Gain compression factor $(\varepsilon)$ is another important parameter of semiconductor laser diodes, which its value can affect on the RIN level. It mainly depends on several mechanisms such as spatial hole burning, spectral hole burning, and other nonlinearities [14]. Figure 6 shows the effect of $\varepsilon$ on the RIN characteristics. As shown, by increasing $\varepsilon$ from $1 \times 10^{-17}$ to $5 \times 10^{-17}$, the peak RIN level has decreased from -118 to $138.3 \mathrm{~dB} / \mathrm{Hz}$, which is due to increment of the decay rate of relaxation oscillation that depends on $\varepsilon$. On the other hand, the ROF value is not affected and maintained at around $1.9 \mathrm{GHz}$.

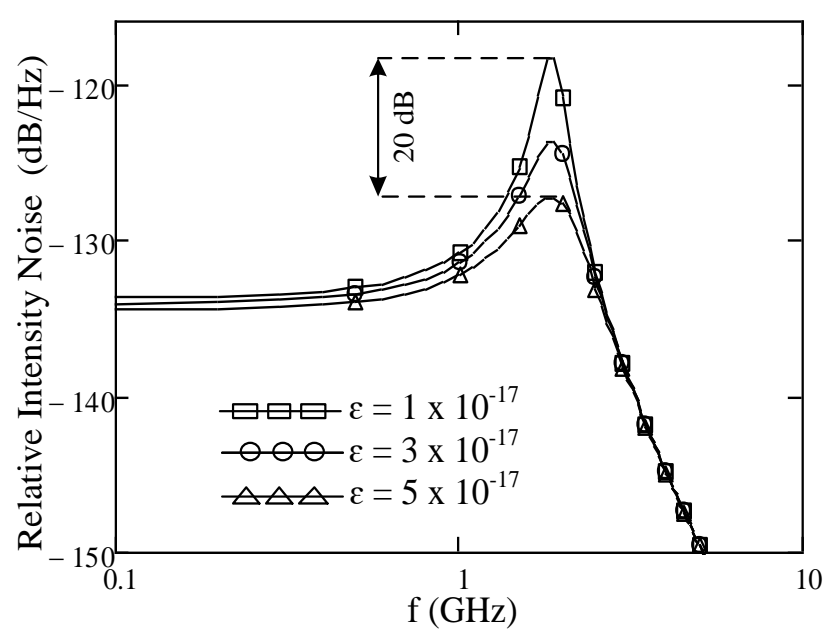

Fig. 6 Effect of nonlinear gain compression factor $(\varepsilon)$ on the DFB RIN spectra

Spontaneous emission factor $\left(\beta_{s p}\right)$ represents one of the affecting parameters on the dynamic behavior for the semiconductor laser diodes.
Where, when the process of recombination is occur to supply a photon into the lasing mode; the rate of the spontaneous emitted photons will increase [6]. During this period, the fluctuations inside the active-layer cavity will increase until the photons reach its steady state value. This process leads to increase the peak RIN level as can be seen in Fig. 7. Also, result shows by increasing $\beta_{s p}$ from $1 \times 10^{-5}$ to $15 \times 10^{-5}$, the peak RIN level has increases by about $4 \mathrm{~dB}$ and ROF has shifted back toward low frequency by around $0.8 \mathrm{GHz}$. For ROF, this result is not consistent with what is given in [6].

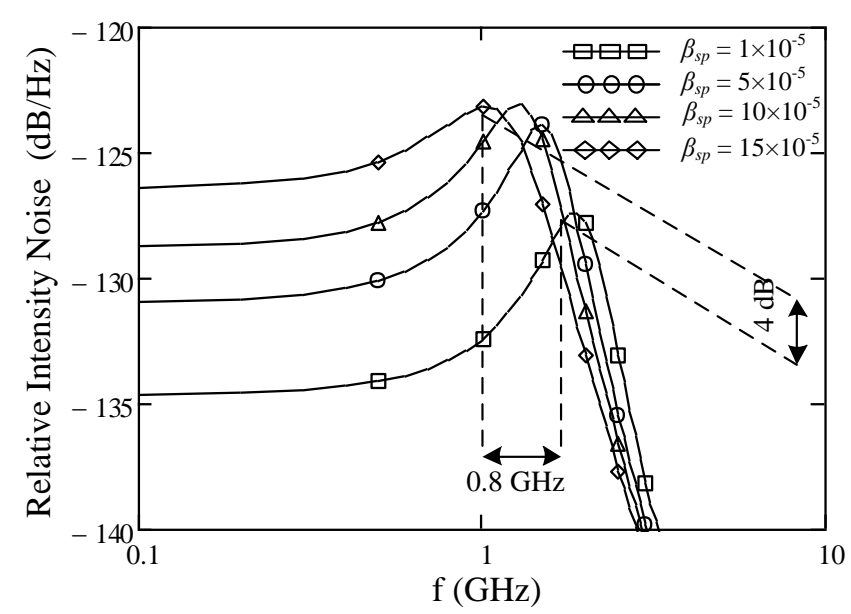

Fig. 7 Effect of $\beta_{s p}$ on RIN spectra of DFB laser

Based on the above discussions, the following values are found to be the best for low RIN level operation, and therefore proposed as guidelines for designers and engineers who are interested in this type of lasers, which are listed in Table II.

TABLE II

BEST VALUES FOR LOW RIN LEVEL

\begin{tabular}{|l|l|}
\hline \multicolumn{1}{|c|}{ Parameter } & \multicolumn{1}{|c|}{ Value } \\
\hline $\begin{array}{l}\text { Active-layer cavity volume } \\
(V)\end{array}$ & $\leq 1.6^{\times 10^{-10} \mathrm{~cm}^{3}}$ \\
\hline Temperature of operation $(T)$ & $T_{o}+20^{\circ} \mathrm{C}$ \\
\hline Injection current $\left(I_{\text {inj }}\right)$ & $\geq 3 I_{\text {th }}$ \\
\hline Gain compression factor $(\varepsilon)$ & $\geq 5 \times 10^{-17}$ \\
\hline $\begin{array}{l}\text { Spontaneous emission factor } \\
\left(\beta_{s p}\right)\end{array}$ & $<5 \times 10^{-5}$ \\
\hline
\end{tabular}


VII. CONCLUSION

Results show the value of the injected current ( $\left.I_{i n j}\right)$ effect significantly on the RIN level, where by increasing $I_{i n j}$ from $1.3 I_{t h}$ to $4 I_{t h}$, the peak RIN level has reduced by an approximately $40 \mathrm{~dB} / \mathrm{Hz}$. In contrast, temperature variations leads to increase the RIN level by the rate of $0.2 \mathrm{~dB} /{ }^{\circ} \mathrm{C}$ while ROF has reduced by the rate of 0.018 $\mathrm{GHz} /{ }^{\circ} \mathrm{C}$. However, temperature effect can be minimized by increasing the $I_{i n j}$ value. Conversely, there is no highly significant effect on RIN level with spontaneous emission factor varying, while increasing the gain compression factor leads to reduce the peak RIN level with constant ROF point.

\section{REFERENCES}

[1] T. Rahman, D. Rafique, A. Napoli, E. Man, B. Spinnler, M. Bohn, C. Okonkwo, A. M. J. Koonen, H. Waardt,'Ultralong Haul 1.28Tb/s PM-16QAM WDM Transmission Employing Hybrid Amplification", J. Lightwave Technolo., Vo. 33, pp. 1794 - 1804, 2015.

[2] D. Adhikari, S. Kadam and Tanushree, "Performance analysis of an $8 \times 8$ STBC coded MIMO system for high rate data transfer", Inte. Conf. on Information Processing (ICIP), pp. 610-614, 2015

[3] N. Hassan, C. Yuen and M. B. Atique,"Tradeoff in delay, cost, and quality in data transmission over TV white spaces", IEEE Inte. Conf. on Communications (ICC), pp. 1-6, 2016

[4] H. K. Hisham, G. A. Mahdiraji, A. F. Abas, M. A. Mahdi, F. R. Mahamd Adikan "Characterization of Turn-On Time Delay in a Fiber Grating Fabry-Perot Lasers" IEEE Photon. Journal, vol. 4, pp 1662-1678, 2012.

[5] J.Li, H. Wang, X. Chen, Z. Yin, Y. Shi, Y. $\mathrm{Lu}, \mathrm{Y}$. Dai and H. Zhu, "Experimental demonstration of distributed feedback semiconductor lasers based on reconstructionequivalent-chirp technology", Opt. Express, Vol. 17, pp5240-5245, 2009.

[6] H. K. Hisham, A. F. Abas, G. A. Mahdiraji, M. A. Mahdi, A. S. Muhammad Noor, "Relative Intensity Noise Reduction by Optimizing Fiber Grating Fabry-Perot Laser
Parameters," IEEE J. Quantum Electron., vol. 48, pp. 385-393, 2012,

[7] I. Fatadin, D. Ives, and M. Wicks," Numerical simulation of intensity and phase noise from extracted parameters for CW DFB lasers," IEEE J. Quantum Electron., vol. 42, pp. 934-941, 2006.

[8] M. Ahmad, M. Yamada, and M. Saito," Numerical modeling of intensity and phase noise in semiconductor lasers," IEEE J. Quantum Electron., vol. 37, pp. 1600-1610, 2001.

[9]J. Liu, W. Chen, J. Guo,; W. Wang, X. Zhou and N. Zhu, "Narrow linewidth distributed-feedback laser with low relative intensity noise", $14^{\text {th }}$ Int. Conf. optical communications and Networking (ICOCN), 2015.

[10] M. Faugeron; M.ël Tran; O. Parillaud; M. Chtioui; Y. Robert; E. Vinet; A. Enard; J. Jacquet; F. Dijk, "High-Power Tunable Dilute Mode DFB Laser With Low RIN and Narrow Linewidth", IEEE Photonics Technology Letters, vol. 25, 2013.

[11]M. Faugeron; M.1 Tran; O. Parillaud; M. Chtioui; Y. Robert; E. Vinet; A. Enard; J. Jacquet; F. Dijk, "High-Power, Low RIN 1.55$\mu \mathrm{m}$ Directly Modulated DFB Lasers for Analog Signal Transmission", IEEE Photonics Technology Letters, vol. 24, 2012.

[12] M. Ming and K. Liu, Principle and applications of optical communication, The McGraw-Hill: USA, 1996.

[13] G. P. Agrawal and N. K. Dutta, LongWavelength Semiconductor lasers. New York: van Nostrand Reinhold, 1986.

[14] H.K. Hisham, A.F. Abas, G.A. Mahdiraji, M.A. Mahdi and A.S. Muhammad Noor," Characterization of phase noise in a singlemode fiber grating Fabry-Perot laser", J. Modern Optics, pp. 1-9, 2011. 